\title{
Médiévales
}

Langues, Textes, Histoire

53 | automne 2007

La nature en partage

\section{«Paris 1377-78 ». Un lieu de pouvoir et sa visibilité entre Moyen Âge et temps présent}

Traduit de l'allemand par Pierre Monnet

Paris 1377-78. Places of Power and the Problems of Visibility between the Middle Ages and Present Time.

\section{Bernd Carqué}

\section{OpenEdition}

\section{Journals}

Édition électronique

URL : https://journals.openedition.org/medievales/4292

DOI : $10.4000 /$ medievales.4292

ISSN : $1777-5892$

\section{Éditeur}

Presses universitaires de Vincennes

\section{Édition imprimée}

Date de publication : 1 décembre 2007

Pagination : 123-142

ISBN : 978-2-84292-211-5

ISSN : 0751-2708

Référence électronique

Bernd Carqué, « "Paris 1377-78 ». Un lieu de pouvoir et sa visibilité entre Moyen Âge et temps présent », Médiévales [En ligne], 53 | automne 2007, mis en ligne le 17 décembre 2009, consulté le 22 avril 2022. URL : http://journals.openedition.org/medievales/4292 ; DOI : https://doi.org/10.4000/ medievales.4292 


\title{
Bernd CARQUÉ
}

\author{
«PARIS 1377-1378 » \\ UN LIEU DE POUVOIR ET SA VISIBILITÉ \\ ENTRE MOYEN ÂGE ET TEMPS PRÉSENT
}

On serait en droit d'attendre du regard de l'historien de l'art s'attachant, pour le cas parisien, aux «images d'une capitale au Moyen Âge ${ }^{1}$ une sorte d'évidence visuelle, puisque l'objet de son enquête annonce en effet la promesse d'une proximité et d'une matérialité des objets. N'est-il pas justement dans la nature de l'histoire de l'art de quêter l'essence des médias par lesquels Paris, au Moyen Âge, s'est présentée comme une capitale, comme un lieu de pouvoir? Il semble par conséquent que le sujet des propos qui vont suivre doive nécessairement résider dans les vestiges matériels du passé, dans l'aspect figuré des productions architecturales et iconographiques par lesquelles le pouvoir a revêtu une forme visible.

Pourtant, cette impression est trompeuse. Car une histoire de la représentation monumentale du pouvoir dans le Paris de la fin du Moyen $\hat{A g e}^{2}$ inclut précisément et obligatoirement les lacunes mêmes de ce "patri-

1. Cet article reprend le texte d'une conférence prononcée lors des rencontres du « Jeudi » de l'Institut Historique Allemand de Paris en décembre 2004. Le thème de l'atelier était consacré à Paris - images d'une capitale du Moyen Âge. Pour la présente publication, la forme de l'exposé a largement été maintenue, les notes bibliographiques les plus importantes ont été ajoutées. L'auteur remercie très cordialement Pierre Monnet pour sa traduction. Une version remaniée et considérablement étendue du texte paraîtra en allemand sous le titre "Orte und Zeichen der Herrschaft im spätmittelalterlichen Paris. Probleme der Sichtbarkeit um 1400 und heute », dans C. EHLERS dir., Places of Power - Lieux de pouvoir - Orte der Herrschaft, Göttingen, à paraître.

2. Pour l'histoire de Paris à la fin du Moyen Âge, consulter en premier lieu : R. CAZELLES, Nouvelle histoire de Paris. De la fin du règne de Philippe Auguste à la mort de Charles V, 1223-1380, Paris, 1972 ; J. FAVIER, Nouvelle histoire de Paris. Paris au XV siècle, 1380-1500, Paris, 1974 ; ID., Paris. Deux mille ans d'histoire, Paris, 1997: sur Paris comme résidence royale notamment les p. 78-82, 271-278 et 767-795. Voir aussi Fr. Pleybert dir., Paris et Charles V. Arts et architecture, Paris, 2001 ; B. Bove, «Les palais royaux à Paris au Moyen $\hat{\mathrm{A} g e}\left(\mathrm{XI}^{\mathrm{e}}-\mathrm{XV}^{\mathrm{e}} \mathrm{s}\right.$.) », dans M.-Fr. AuZÉPY et J. ConnetTe dir., Palais et pouvoir. De Constantinople à Versailles, Saint-Denis, 2003, p. 45-80 ; S. Roux, Paris au Moyen Âge, Paris, 2003. 
moine » et raconte l'histoire de ses pertes substantielles. Là où une histoire de la civilisation - inscrite dans la tradition de Voltaire et de Condorcet raconte depuis la publication de l'Histoire physique, civile et morale de Paris par Jacques-Antoine Dulaure ${ }^{3}$ le développement d'un progrès irrésistible; là où cette histoire célèbre, avec la «Transformation de Paris » ordonnée par Napoléon III et accomplie par le préfet de la Seine Georges-Eugène Haussmann, le triomphe remporté sur la ville médiévale et ses conditions néfastes de vie ; là aussi où, du haut de la «Tour de trois cents mètres » de Gustave Eiffel, elle chante le mythe de la Modernité engendrée par la Révolution; en tous ces lieux, l'archéologie médiévale raconte une histoire de disparition et de destruction. Elle fait le récit de la démolition du Louvre médiéval, entamée sous François ${ }^{\mathrm{I} r}$, du démantèlement révolutionnaire de la Bastille ou de l'effrayant "éventrement du vieux Paris », opéré sous Haussmann ${ }^{4}$. Les images renvoient également l'écho de semblables motifs narratifs concurrents. La photographie - l'incarnation de la modernité picturale - met en vue les grandes constructions en fer comme la «Galerie des Machines » ou la Tour Eiffel, témoin monumental par excellence de l'idéologie du progrès propagée par la Troisième République. En même temps, les images traditionnelles de la gravure ou de l'eau-forte retiennent les ruines pittoresques du vieux Paris à l'exemple des vedute romantiques.

Autrement dit, c'est justement ce qui incarne de manière visible le pouvoir à la fin du Moyen Âge, ce qui l'a aidé, dans le paysage urbain, à acquérir une présence durable - c'est-à-dire les édifices des résidences et des fortifications, les programmes iconographiques de leurs façades et de leurs portes - ce sont tous ces médias qui se soustraient aujourd'hui dans leur plus grande majorité à notre observation directe. En lieu et place, l'historien de l'art se voit confronté à des artefacts de compensation aux formes multiples, par le truchement desquelles la visibilité perdue de l'histoire doit être reconquise. Avant même que l'archéologie urbaine n'émerge dans la seconde moitié du XIX ${ }^{\mathrm{e}}$ siècle sur le socle de l'ample documentation des sources écrites et des vestiges matériels, et avant même que le baron Haussmann n'entreprenne d'éditer - dans une singulière dialectique de la destruction et de la conservation - sa monumentale Histoire générale de Paris ${ }^{5}$, c'est l'imagination du passé qui surgit à la place de son présent matériel.

Pour rendre une objectivation allégorique de la connexion de tous les domaines de la vie sociale qui éclate au seuil de la Renaissance, Victor Hugo, dans Notre-Dame de Paris, ne se contente pas d'en appeler à la cathé-

3. J.-A. Dulaure, Histoire physique, civile et morale de Paris depuis les premiers temps historiques jusqu'à nos jours, Paris, 8 vol., 1821-1825.

4. Pour une vue d'ensemble des destructions voir L. Réau, Histoire du Vandalisme. Les monuments détruits de l'art français, éd. augmentée par M. Fleury et G.-M. LEProuX, Paris, 1994 ; P. LÉON, La Vie des monuments français. Destruction, restauration, Paris, 1951.

5. Voir en particulier A. BERTy, H. Legrand et L.-M. Tisserand, Topographie historique du vieux Paris, Paris, 6 vol., 1866-1897. 
drale médiévale. Il invoque aussi, et avant tout, l'esprit du Palais de la Cité. Dans le chapitre introductif de cette fresque romanesque, chapitre qui, rappelons-le, en porte le nom même, c'est-à-dire la Grand'Salle du Palais, cette dernière est le théâtre de la Fête des fous et de l'élection de leur pape, scènes dont les grotesques uniformisantes veulent exprimer l'irréductible et par essence médiévale totalité de la vie. C'est de manière analogue que, par la suite, les illustrateurs du roman ont recomposé par l'imagination les monuments perdus ${ }^{6}$. Depuis Victor Hugo, la Grand'Salle royale de Philippe le Bel compte, à ce titre, parmi les «lieux de mémoire » majeurs d'une conscience urbaine bourgeoise et républicaine. Viollet-le-Duc, dans son Dictionnaire raisonné de l'architecture l'a fait revivre par les lignes de ses plan et élévation $^{7}$. Pendant l'exposition universelle de 1900, Albert Robida ressuscita la Grand'Salle au milieu d'un «capriccio » architectural qui rassemblait aussi la porte Saint-Michel, une tour du Louvre et d'autres archétypes purement moyenâgeux (fig. 1$)^{8}$.

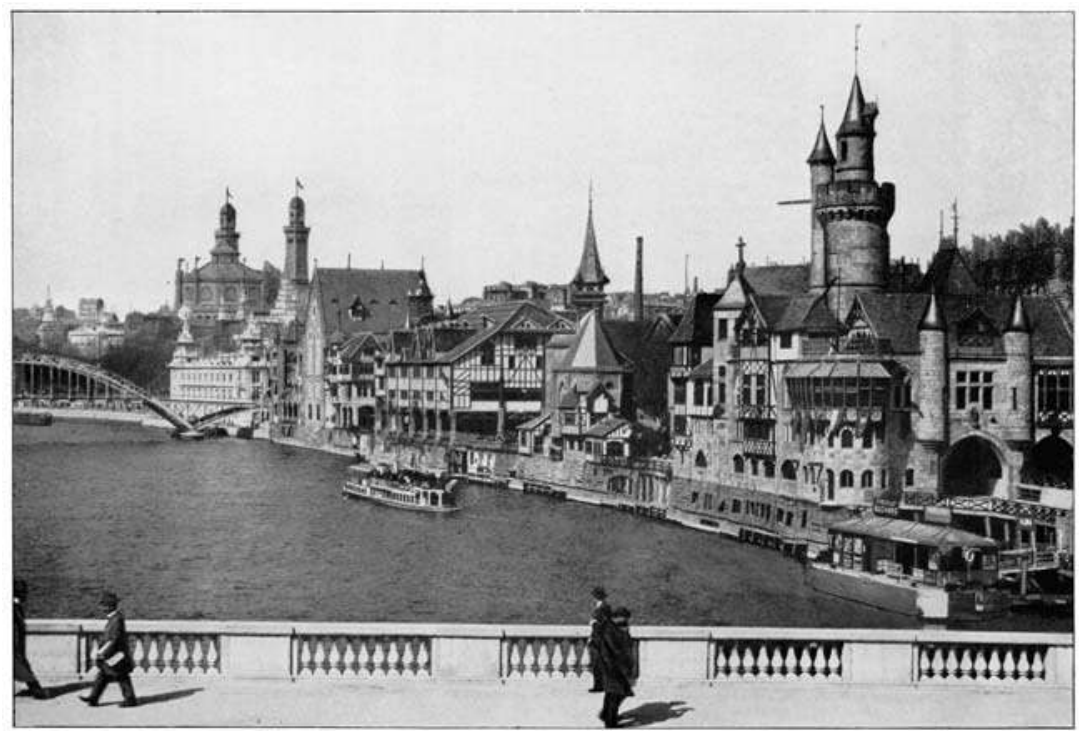

Fig. 1: Le Vieux Paris à l'Exposition universelle de 1900. Archives de l'auteur.

6. V. Hugo, Notre-Dame de Paris. Nouvelle édition illustrée, Paris, 2 vol., 1876-1877, p. 5-23, les xylographies d'après Fédor Hoffbauer se trouvent en p. 7 et 9.

7. E.-E. VIOLLET-Le-Duc, article «Salle», dans ID., Dictionnaire raisonné de l'architecture française du XI ${ }^{e}$ au XVI ${ }^{e}$ siècle, Paris, 10 vol., 1854-1868, vol. VIII, p. 71-95, pour la Grand'Salle voir p. 82-84 avec les fig. 5 (plan) et 6 (élévation).

8. A. RoBIDA, Exposition universelle de 1900. Le Vieux Paris, guide historique, pittoresque et anecdotique, Paris, 1900 ; ID., Exposition universelle de 1900. Le Vieux Paris, études et dessins originaux, Paris, 1900. 
Quant au Louvre, Viollet-le-Duc en donne dans son Dictionnaire une présentation d'architecte rationaliste, de toute la hauteur d'une analyse structurelle à vol d'oiseau (fig. 2) ${ }^{9}$, tandis qu'Alphonse de Neuville, dans une édition illustrée de l'Histoire de France de François Guizot ${ }^{10}$, en projette une image visionnaire avec toute la rhétorique visuelle et les décors mobiles du romantisme (fig. 3). Ainsi le Louvre est-il l'exemple caractéristique d'une forteresse tardo-médiévale bien structurée et en même temps «cette hydre de tours, gardienne géante de Paris » comme Victor Hugo se l'est imaginé ${ }^{11}$. Les statues conservées de Charles $\mathrm{V}$ et de son épouse Jeanne de Bourbon, provenant du portail oriental du Louvre, étaient exposées jusqu'en 1816 au Musée des Monuments français mais signalées comme étant celles de «saint Louis » et de «Marguerite de Provence » ${ }^{12}$. Ce faisant, elles personnifiaient ce Moyen Âge chrétien qu'Alexandre Lenoir avait mis en scène comme un tableau d'époque dans la salle consacrée au XIII ${ }^{e}$ siècle $^{13}$. Dans les années 1870 l'œuvre de Fédor Hoffbauer, Paris à travers les âges, projeta entre autres l'écho visuel de l'Hôtel Saint-Pol, du couvent des Célestins, de l'enceinte de Charles V, de la Bastille et de la porte Saint-Antoine dans la vue cavalière des vedute ${ }^{14}$. Cependant, on constate que l'enceinte perdue prend pour l'occasion une forme que les architectes et restaurateurs du XIX ${ }^{\mathrm{e}}$ siècle ont imaginée et réalisée pour Avignon ou Carcassonne ${ }^{15}$.

Faisons bref : la question de l'aspect concret des lieux et des signes du pouvoir dans le Paris tardo-médiéval s'avère particulièrement épineuse. Elle est en effet tributaire d'une transmission problématique dans laquelle les monuments en question ne sont plus présents qu'en images et n'apparaissent plus dans ces dernières qu'avec une variabilité kaléidoscopique. Des schémas de vision et d'interprétation multiformes et temporellement contingents ont produit des visualisations non moins multiformes. Par leur biais nous sommes

9. E.-E. Viollet-Le-Duc, article «Château », dans ID., Dictionnaire raisonné..., op. cit., vol. III, p. 58-191, pour le Louvre, p. 134-140.

10. Fr. Guizot, Histoire de France depuis les temps les plus reculés jusqu'en 1789, racontée à mes petits-enfants, Paris, 5 vol., 1873-1876, vol. 2, p. 141.

11. V. Hugo, Notre-Dame de Paris, op. cit., vol. 1, p. 170.

12. Voir à ce sujet (avec bibliographie) Fr. BAron, Musée du Louvre, Département des sculptures du Moyen Âge, de la Renaissance et des temps modernes. Sculptures françaises I: Moyen Âge, Paris, 1996, p. 150.

13. Vues pittoresques et perspectives des salles du Musée des Monumens François, et des principaux ouvrages d'architecture, de sculpture et de peinture sur verre, qu'elles renferment. Gravées [...] par Réville et Lavallée. Avec un texte explicatif par de Roquefort, Paris, 1816, planche I.

14. F. HoffBAuer, Paris à travers les âges. Aspects successifs des monuments et quartiers historiques de Paris depuis le XIII e siècle jusqu'à nos jours, fidèlement restitués d'après les documents authentiques, Paris, par livraisons successives de 1875 à 1882, reprint Paris, 2001, p. 551 (fig. 1) et planche suivant la p. 552.

15. E.-E. Viollet-Le-Duc, La cité de Carcassonne (Aude), Paris, 1858 (éd. augmentée en 1878) ; Viollet-le-Duc, Paris, 1980 (catalogue de l'exposition de Paris), p. 114-123. 


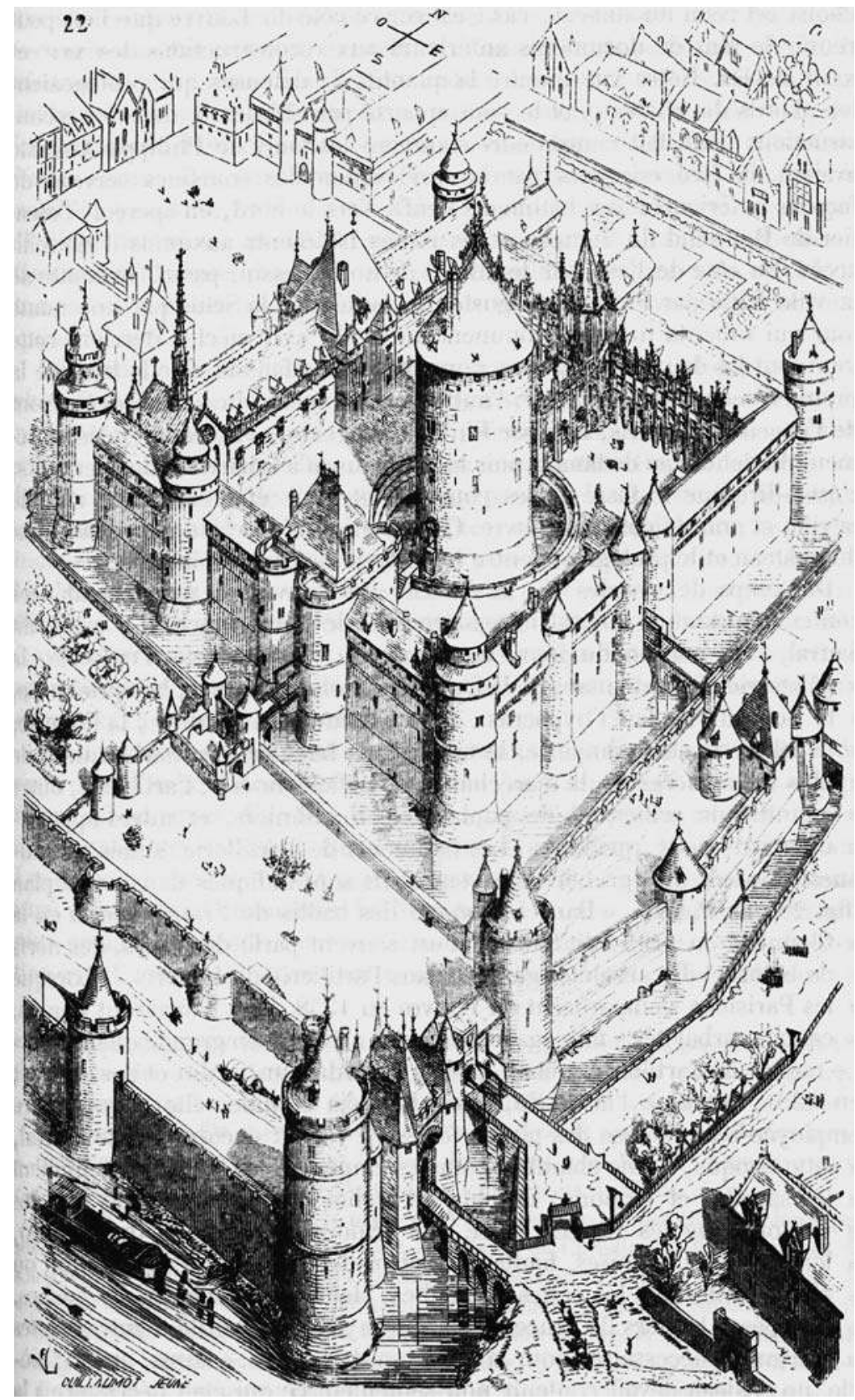

Fig. 2 : Le château du Louvre d'après Viollet-le-Duc. Archives de l'auteur. 


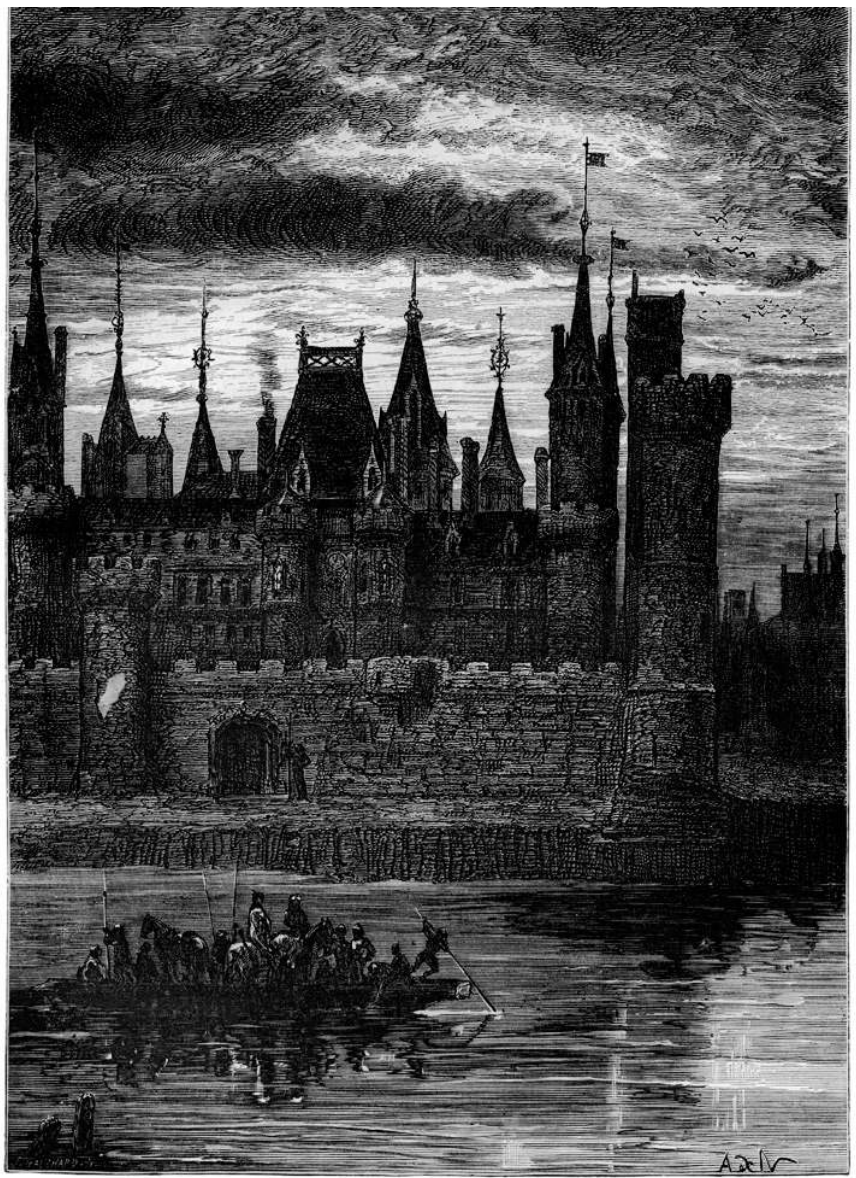

Fig. 3 : Le château du Louvre d'après Alphonse de Neuville. Archives de l'auteur.

en fait exhortés à revenir en premier lieu sur les conditions de notre propre perception et à nous demander sur quoi repose notre savoir au cas par cas et à quelles sources s'alimentent nos conceptions d'une représentation de la monumentalité du pouvoir.

Selon toute apparence, on ne peut tirer de certitude relative que dans le cas de la cour royale. Car ce qui n'est qu'exceptionnellement possible dans le cas des institutions princières, ecclésiastiques, universitaires ou communales semble constituer un correctif de taille. En effet, dans son effort de restitution de la visibilité perdue des monuments, la recherche peut non seulement s'appuyer sur ce qui a été sauvegardé ou bien sur ce que l'archéologie a exhumé, mais elle peut également mobiliser le secours des témoignages iconographiques du temps. 
Les miniatures du calendrier des Très Riches Heures du duc de Berry ${ }^{16}$ (vers 1415 et 1438-1442) en constituent l'un des exemples les plus célèbres. La miniature du mois de juillet, comportant le Palais de la Cité ${ }^{17}$, propulse dans l'image même le cœur fonctionnel du royaume, le siège politique et administratif de la couronne ${ }^{18}$. La représentation, grâce au double pignon si marquant de la Grand'Salle, renvoie à l'espace central où se jouent la mise en scène et l'action du pouvoir royal, au lieu où se déroulent les actes de justice, les réceptions d'ambassades, les festivités de cour. Avec la SainteChapelle, c'est-à-dire la chapelle du chapitre collégial royal, l'image désigne le lieu de conservation des reliques les plus importantes de la couronne. $\mathrm{Au}$ centre de la miniature du mois d'octobre, c'est le Louvre qui est montré ${ }^{19}$, c'est-à-dire le siège le plus notable de la maison et de la cour du roi depuis Charles $\mathrm{V}^{20}$. De la sorte, tout comme l'assure cet édifice entre la scène des semailles au premier plan et les données astrologiques contenues dans le tympan en couronne, le Palais de la Cité sert également d'intermédiaire, dans l'image, entre l'espace social des paysans et l'espace cosmique du calendrier de l'année. Sur la miniature du mois de décembre ${ }^{21}$, le bois de Vincennes, lieu scénique de la chasse, est surplombé par les tours et le donjon du château. Ce lieu du pouvoir royal est lui aussi - comme les châteaux ducaux exposé à travers l'ordre visuel spécifique de la miniature comme un centre, tant physique que métaphysique, de l'ordre politique et social.

Cependant, les monuments en question ne désignent pas seulement le centre idéel de l'espace socio-politique, mais ils sont également insérés dans les espaces de l'économie divine du Salut - ainsi par exemple du Livre d'heures (vers 1452-1460) d'Étienne Chevalier, le trésorier de France sous Charles VII et Louis XI, dans lequel le donjon du château de Vincennes apparaît à l'arrière-plan de la miniature décorant l'office des morts et ren-

16. Tout dernièrement: Les Très Riches Heures du duc de Berry et l'enluminure en France au début $d u X^{e}$ siècle, Paris, 2004 (catalogue de l'exposition de Chantilly).

17. Chantilly, Musée Condé, ms. $65, \mathrm{f}^{\mathrm{0}} 6 \mathrm{v}^{\circ}$.

18. Les études fondamentales sur le Palais de la Cité sont celles de J. GuÉrout, « Le Palais de la Cité à Paris des origines à 1417. Essai topographique et archéologique », Mémoires de la Fédération des Sociétés historiques et archéologiques de Paris et de l'Ile-de-France 1, 1949, p. 57-212; 2, 1950, p. 21-204; 3, 1951, p. 7-101. ID., "L'hôtel du roi au palais de la Cité à Paris sous Jean II et Charles V », dans J. Chapelot et É. Lalou dir., Vincennes aux origines de l'État moderne, Paris, 1996, p. 219-288 ; voir aussi M. T. DAvIS, «Desespoir, Esperance, and Douce France: The New Palace, Paris, and the Royal State », dans M. BenT et A. WatheY dir., Fauvel Studies, Oxford, 1998, p. 187-213.

19. Chantilly, Musée Condé, ms. $65, \mathrm{f}^{\mathrm{o}} 10 \mathrm{v}^{\circ}$.

20. Sur l'histoire du Louvre médiéval, voir A. Berty, H. Legrand, L.-M. Tisserand, Topographie historique du vieux Paris, op. cit., vol. 1, p. 123-199, et vol. 2, p. 109-168; Le Louvre des Rois. Les fouilles de la Cour Carrée (= Archéologia. Dossiers Histoire et archéologie 110, 1986) ; M. Fleury et V. Kruta, Le Château du Louvre, Paris, 1989, p. 3-80 ; M. Whiteley, «Le Louvre de Charles V. Dispositions et fonctions d'une résidence royale », Revue de l'art, 97, 1992, p. 60-75.

21. Chantilly, Musée Condé, ms. $65, \mathrm{f}^{\mathrm{o}} 12 \mathrm{v}^{\mathrm{o}}$. 
voyant, ce faisant, à la richesse perdue de Job ${ }^{22}$. Que la Sainte-Chapelle, dans le Livre d'heures d'Étienne Chevalier et plus singulièrement dans le contexte précis du Portement de la Croix (fig. 4) ${ }^{23}$, soit représentée avec une telle prégnance se laisse aisément comprendre si l'on songe qu'elle fut construite entre 1241 et 1248, sur ordre de Louis IX, pour servir de reliquaire monumental aux trésors de la Passion acquis à Constantinople.

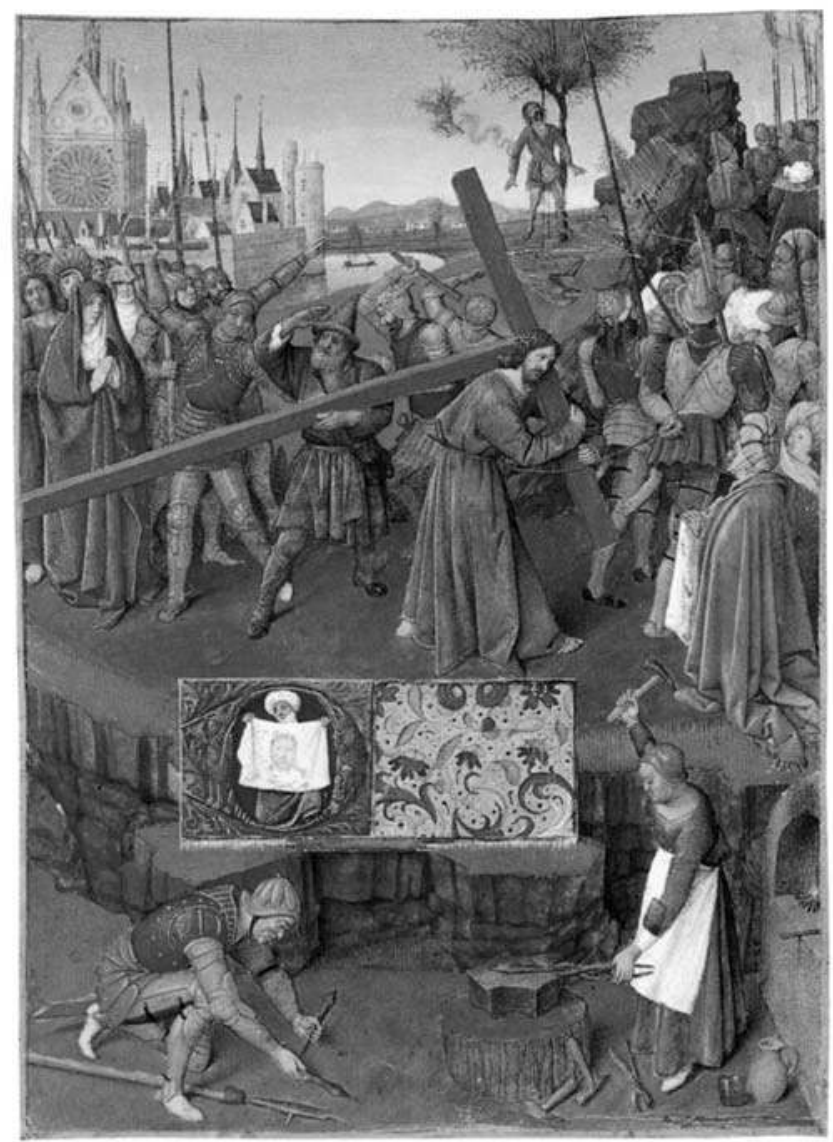

Fig. 4 : «Le Portement de Croix » dans le Livre d'heures d'Étienne Chevalier. Reproduit dans François Avril (sous la direction de), Jean Fouquet. Peintre et enlumineur du XV siècle, Paris, 2003.

22. « Job et ses amis », folio détaché ( $\mathrm{n}^{\circ} 27$ d'après Nicole Reynaud), conservé au musée Condé de Chantilly ; voir Fr. AvrIL dir., Jean Fouquet. Peintre et enlumineur du XV siècle, Paris, 2003 (catalogue de l'exposition de Paris), n 24, p. 193-217.

23. «Le Portement de Croix », folio détaché ( $n^{\circ} 12$ d'après Nicole Reynaud), conservé au musée Condé de Chantilly. 
Dans ce type d'images, les cadres spatiaux de la vie des commanditaires et des contemplateurs des milieux de cour se révèlent comme recouverts jusqu'à l'identification par les espaces sacrés du récit biblique. Ces entrelacs, cette compénétration du profane et du sacré revêtaient une signification toute particulière dans l'entourage de la famille royale, à la mesure du rôle central que pouvaient y jouer les conceptions de l'élection divine et de la sainteté du sang de la lignée.

Des miniatures comme celles-ci montrent bien que, entre l'ordre visuel de l'image, d'un côté, et l'ordre sacré et social, de l'autre, l'on pouvait établir des relations symboliques. Ces dernières ne sont pas sans conséquences sur la valeur documentaire des images. Car l'objet de la représentation n'est pas l'édifice lui-même - au sens, si l'on veut, d'un portrait architectural authentique - mais la signification matérialisée, c'est-à-dire objectivée par son truchement. Les édifices en question, dans l'image, possèdent avant tout une fonction symbolique. C'est la raison pour laquelle les modèles de perception et d'interprétation issus de la théorie politique et de la théologie l'emportent toujours sur un certain mode naturaliste de la représentation.

La confrontation de plusieurs vues atteste que l'on se contentait d'un nombre limité de caractéristiques monumentales qui permettaient de les reconnaître. Il en résulte que les représentations figurées ne peuvent guère délivrer que des informations générales sur l'apparence ancienne des lieux et des signes du pouvoir. Par conséquent, quand le tertium comparationis, c'est-à-dire la forme d'origine du bâtiment, n'a pas été conservé, il n'est tout simplement pas possible d'avancer une quelconque conclusion quant à la ressemblance réelle de la représentation. À l'instar des productions iconographiques imaginaires de la modernité, les représentations de l'époque même ne nous donnent pas à voir les châteaux et les résidences autrement que comme des objets relationnels - tributaires de modes variables de perception et de compréhension autant que de modalités correspondantes de visualisation.

De surcroît, les images démontrent bien que les monuments représentés ne possédaient aucune signification absolue et définitivement fixée. Car elles figurent des lieux et des signes du pouvoir exposés dans des contextes, des situations et des réceptions chaque fois différents. Pour ne prendre qu'un exemple, Jean Fouquet, dans un manuscrit enluminé vers 1455-1460 des Grandes Chroniques de France, a restitué visuellement la porte Saint-Denis, porte d'entrée de Charles V après le sacre de Reims (fig. 5) ${ }^{24}$. Selon une continuité topographique bien précise apparaissent successivement, dans le prolongement de l'enceinte, la porte Saint-Martin, le Temple et, à l'arrièreplan, la Bastille. Mais l'enlumineur flamand d'un manuscrit des Chroniques de Jean Froissart a procédé différemment pour dépeindre, vers 1470, l'entrée

24. Paris, BnF, ms. fr. $6465, \mathrm{f}^{\mathrm{0}} 417$; voir Fr. AvrIL dir, Jean Fouquet, op. cit., $\mathrm{n}^{\circ} 26$, p. 219-248. 


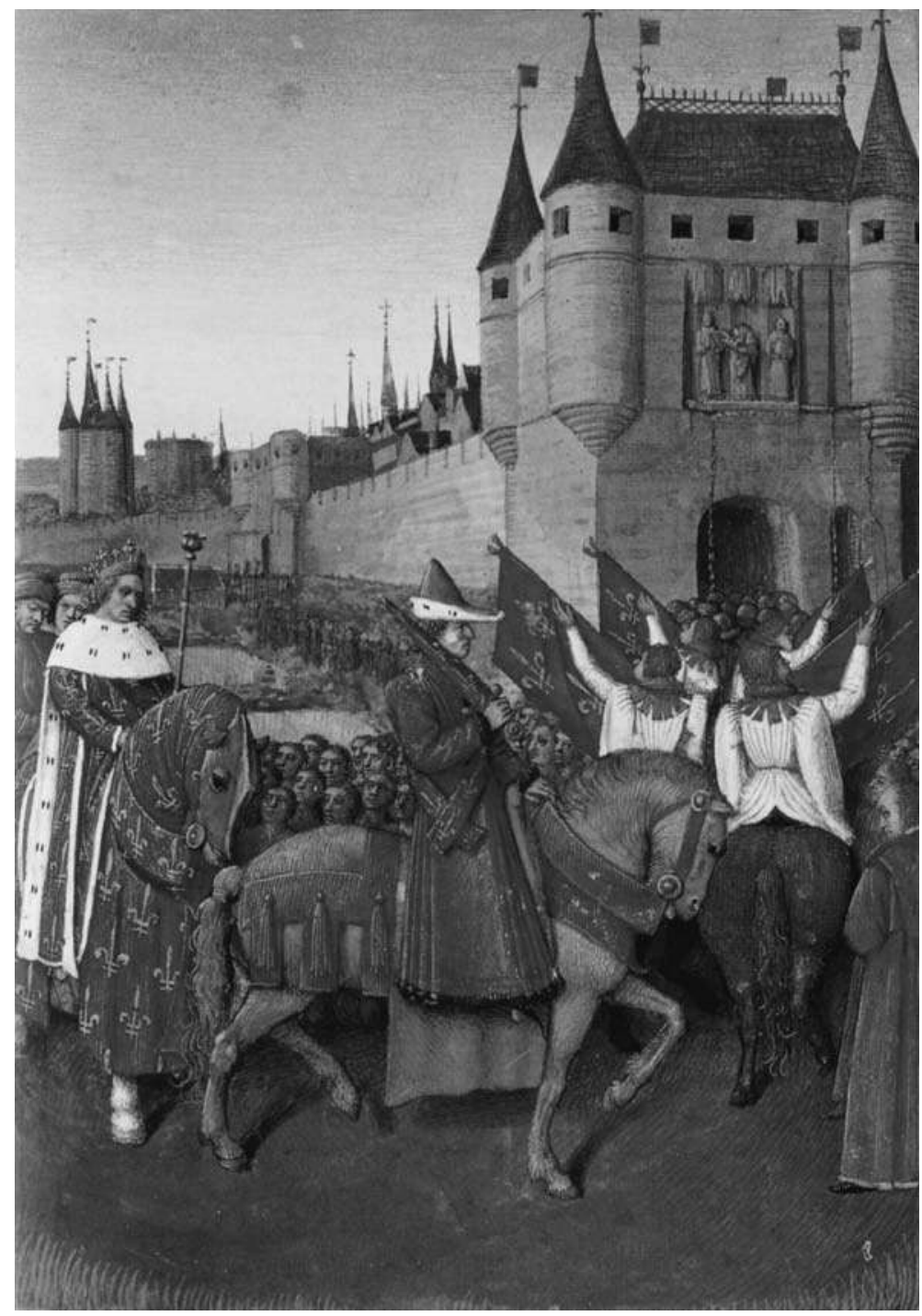

Fig. 5 : «Entrée de Charles V à Paris après le sacre de Reims » dans les Grandes Chroniques de France enluminées par Jean Fouquet.

Reproduit dans François Avril (sous la direction de),

Jean Fouquet. Peintre et enlumineur du XV siècle, Paris, 2003. 
d'Isabeau de Bavière en 1389 (fig. 6) ${ }^{25}$. Il situe dès la porte Saint-Denis l'accueil postérieur rendu par l'évêque de Paris et, ce faisant, comprime plusieurs édifices en un seul panorama symbolique de la ville : à savoir, et selon toute probabilité, le portail oriental du Louvre, la tour de l'Horloge ou la Sainte-Chapelle, lieu du sacre à venir.

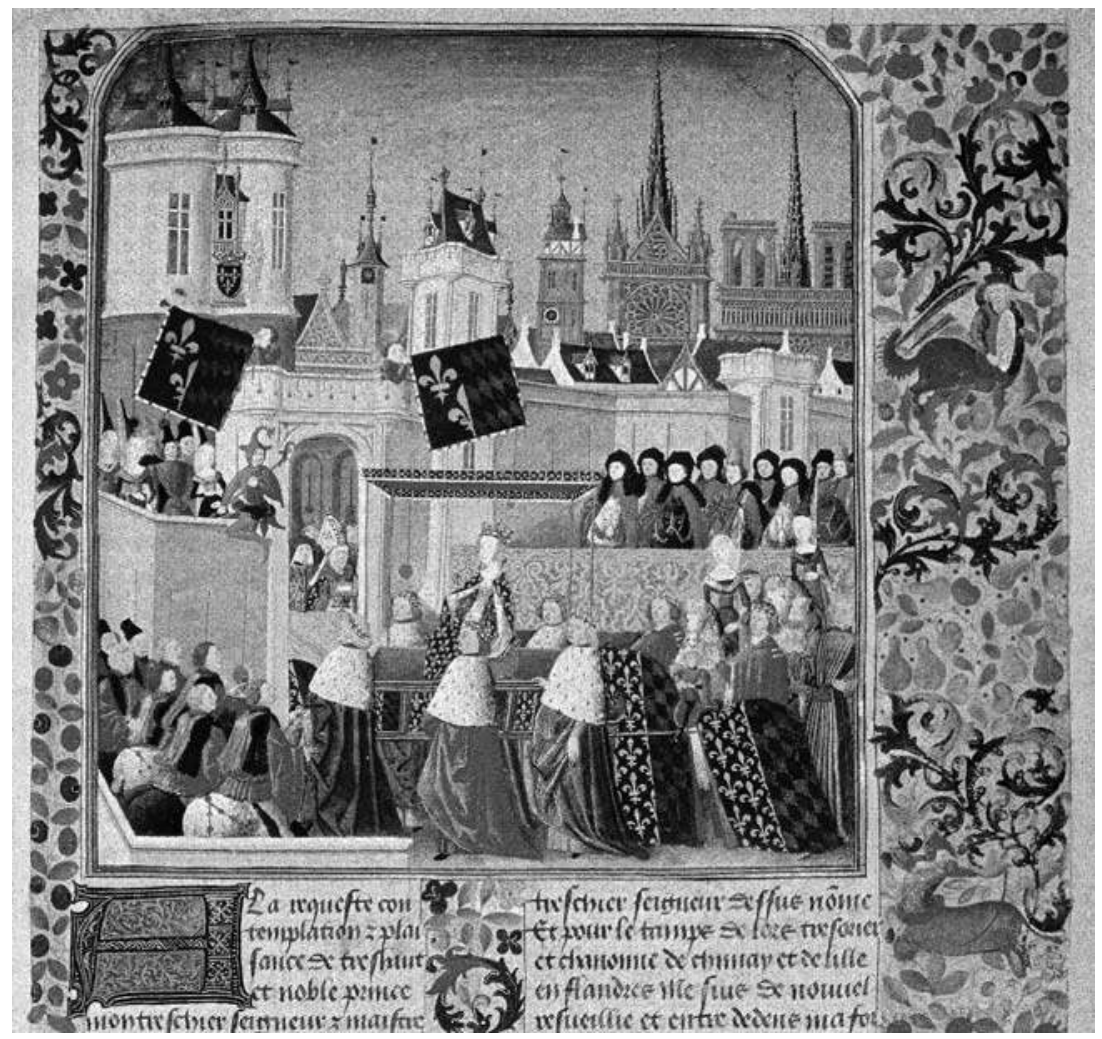

Fig. 6: «Entrée d'Isabeau de Bavière en 1389» dans un manuscrit flamand des Chroniques de Jean Froissart. Reproduit dans Laetitia Le Guay, Les princes de Bourgogne lecteurs de Froissart, Turnhout, 1998.

De telles représentations prouvent bien que les productions monumentales et iconographiques étaient perçues dans le contexte de pratiques sociopolitiques précises et sur un mode spécifique. C'est une invitation à penser que la portée respective des lieux et des signes du pouvoir ne se déployait

25. Londres, British Library, ms. Harley 4379, fo 3 ; L. LE GuAY, Les princes de Bourgogne lecteurs de Froissart, Turnhout, 1998, p. 31-38, 72 sq. et 180 sq. ; M. Whiteley, « Deux vues de l'hôtel royal de Saint-Pol », Revue de l'art, 128, 2000, p. 49-53. 
avec davantage de précision que par le biais des actions accomplies en présence des monuments, et que par conséquent leurs significations ne se livraient pas par elles-mêmes mais étaient produites et concrétisées en situation. Il convient d'en déduire que l'on ne peut capter d'emblée le "sens» ni à partir de la simple lecture des propriétés formelles des monuments ni des contenus figurés des images. Ce «sens » doit bien plutôt être entendu comme un attribut dépendant d'un contexte, et, comme tel, soumis à variation.

C'est pourquoi il ne suffit pas de procéder par le questionnement des formes externes et matérielles, c'est-à-dire par une approche fondée sur une typologie monumentale et sur une iconographie architecturale de phénomènes formels interprétables. Puisque les lieux et les signes du pouvoir, à travers les supports imagés de la fin du Moyen Âge, ont été majoritairement représentés au sein de contextes cérémoniellement structurés, il convient bien davantage d'interroger plus précisément les conditions de perception qui y ont présidé. En raison de la situation documentaire, ce sont avant tout les formes de réception d'un espace public représentatif qui nous paraissent les plus aisées à cerner.

Sous cet angle, la visite de l'empereur Charles IV à la cour de son neveu, à Paris, au cours de l'hiver 1377-1378, nous paraît particulièrement révélatrice. Cet événement que l'historiographie du temps a rapporté avec force détails ${ }^{26}$ a toujours fait l'objet d'une attention soutenue de la part des chercheurs ${ }^{27}$ et a été tout récemment revisité dans la perspective plus particulière de la visualité/visualisation du pouvoir. En effet, les textes parlent d'une large sémiotisation de ces cadres précis de la vie urbaine et curiale devenus provisoirement des espaces de la théâtralisation et du déroulement du cérémoniel de la visite. Dans le même esprit, la recherche s'est attachée aux systèmes sémiotiques d'une représentation performative du pouvoir, c'est-à-

26. Consulter avant tout R. Delachenal éd., Chronique des règnes de Jean II et de Charles V (Les Grandes Chroniques de France), Paris, 4 vol., 1910-1920, vol. 2, p. 193-277.

27. Entre autres : R. Delachenal, Histoire de Charles V, Paris, 5 vol., 1909-1931, vol. V, p. 61-122 ; H. NeureITHER, Das Bild Karls IV. in der zeitgenössischen französischen Geschichtsschreibung, Heidelberg, 1964, en part. p. 112-147 et 215-232 ; M. THOMAs, « La visite de 1'empereur Charles IV en France, d'après l'exemplaire des Grandes Chroniques exécuté pour le roi Charles V », dans VI. Internationaler Kongre $\beta$ der Bibliophilen. Vorträge, Wien, 1971, S. 8598 ; H. Thomas, «Ein zeitgenössisches Memorandum zum Staatsbesuch Kaiser Karls IV. in Paris », dans W. HAUBRICHS et W. LAUFER dir., Zwischen Saar und Mosel. Festschrift für HansWalter Herrmann zum 65. Geburtstag, Sarrebrück, 1995, p. 99-119 ; Fr. AUTRAND, « Mémoire et cérémonial : la visite de l'empereur Charles IV à Paris en 1378 d'après les Grandes Chroniques de France et Christine de Pizan », dans L. Dulac et B. RibÉmont dir., Une femme de lettres au Moyen Âge. Études autour de Christine de Pizan, Orléans, 1995, p. 91-103 ; A. STRUBEL, «Le chevauchier de Charles V. Christine de Pisan et le spectacle de la majesté royale », dans D. Boutet et J. Verger dir., Penser le pouvoir au Moyen Âge (VIII ${ }^{e} X V^{e}$ s.). Études d'histoire et de littérature offertes à Françoise Autrand, Paris, 2000, p. 385-399. 
dire aux couleurs et aux blasons, aux livrées et aux montures, ou bien à l'ordre protocolaire des cortèges et des places ${ }^{28}$.

En revanche, on n'a prêté pratiquement aucune attention au fait que l'itinéraire de ce voyage peut également s'interpréter, pour ainsi dire, comme un inventaire des espaces de représentation du pouvoir royal, espaces chargés non de signes éphémères mais de signes de présence durable, espaces emplis non des médias mobiles du cérémoniel mais d'édifices et d'images fixes. Leur perception ne fut pas non plus laissée au hasard. C'est le souverain en visite qui a expressément formulé le vœu de les contempler («il demanda à veoir » ainsi qu'on le trouve exprimé à plusieurs reprises dans les Grandes Chroniques de France), vœu exaucé et orchestré par la puissance invitante.

D'abord l'abbaye royale de Saint-Denis ${ }^{29}$ : ici l'empereur rendait visite au lieu de pouvoir le plus éminent en dehors de la ville. Les reliques ${ }^{30}$ constituaient le but déclaré de la demande impériale de contemplation, de dévotion et de contact physique. Armes et inscriptions présentaient sans équivoque aucune les reliquaires comme autant de fondations royales. À l'endroit des « sepultures des roys », Charles IV commémora les liens familiaux étroits qui unissaient les Luxembourg aux Capétiens et aux Valois. Comme l'empereur ne rencontra le roi que sur le chemin qui le conduisait à Paris, il ne put jouir dans l'abbaye même de la faveur qui nous est rapportée par Christine de Pisan dans son Livre des fais et bonnes meurs du sage Roy Charles relatant que, en signe de témoignage particulier d'honneur, Charles V avait coutume de conduire ses hauts visiteurs à Saint-Denis et ouvrait en personne les coffres et armoires afin de montrer à ses invités les reliques, les parements et les insignes du couronnement ${ }^{31}$.

Pour ce qui touche également à l'entrée de l'empereur et au cortège traversant la ville, les Grandes Chroniques mettent expressément en exergue le caractère de visibilité et, de manière pendante, les perceptions et les effets visuels : «c'estoit noble et merveilleuse chose à veoir »; les témoins oculaires « furent moult merveilliez, qui autrefoiz n'avoient veue tele ne si bonne ordenance de tele multitude, si pou de desroy ne de presse ${ }^{32}$. La

28. Pour les cérémonies des entrées en général consulter entre autres: B. GuENÉE et Fr. Lehoux, Les entrées royales françaises de 1328 à 1515, Paris, 1968; L. M. BRYANT, The King and the City in the Parisian Royal Entry Ceremony. Politics, Ritual, and Art in Renaissance, Genève, 1986 ; G. KIPLING, Enter the King. Theatre, Liturgy, and Ritual in the Medieval Civic Triumph, Oxford, 1998 ; G. J. SchEnK, Zeremoniell und Politik. Herrschereinzüge im spätmittelalterlichen Reich, Cologne, 2003 ; E. LecuPPRE-DESJARDIn, La ville des cérémonies. Essai sur la communication politique dans les anciens Pays-Bas bourguignons, Turnhout, 2004 ; J. Hurlbut, "Vive Bourgogne est nostre cry». Ceremonial Entries of Philip the Good and Charles the Bold (1419-1477), Turnhout, à paraitre en 2007.

29. M. FÉlibien, Histoire de l'Abbaye Royale de Saint-Denys en France, Paris, 1706 ; Fr. Autrand dir., Saint-Denis et la royauté. Mélanges offerts à Bernard Guenée, Paris, 1999.

30. Le trésor de Saint-Denis, Paris, 1991 (catalogue de l'exposition de Paris).

31. Christine de Pisan, Le Livre des fais et bonnes meurs du sage Roy Charles, éd. S. Solente, Paris, 2 vol., 1936-1940, vol. 2, p. 87.

32. Chronique des règnes de Jean II et de Charles V, op. cit., vol. 2, p. 222. 
perception impériale était guidée par Charles V lui-même. Parvenu au Palais de la Cité, le roi conduisit son oncle depuis la cour vers la Grand'Salle en empruntant la Galerie Mercière. Le Palais s'ouvrait par les Grands Degrés qui menaient au portail de la Galerie Mercière. Au trumeau du portail dressé par Philippe le Bel, le visiteur pouvait apercevoir une statue montrant ce roi dans son habit du couronnement. ${ }^{33} \mathrm{La}$ Grand'Salle formait le centre fonctionnel du siège décisionnel et administratif de la couronne, la tribune des actes de justice, le théâtre de la réception des ambassades et de l'organisation des fêtes de cour. ${ }^{34}$ Sur la gravure fameuse de Jacques I ${ }^{\text {er }}$ Androuet du Cerceau représentant l'intérieur de la Grand'Salle ${ }^{35}$, on peut reconnaître la série des statues plus grandes que nature des rois «français» depuis Pharamond, dont la confection avait débuté sur l'ordre de Philippe IV. Les inscriptions comportaient pour chacun des rois, à côté de son nom, la durée du règne, l'année de sa mort et le cas échéant la relation de parenté la plus proche. Dans son éloge urbain Tractatus de laudibus parisius de 1323, Jean de Jandun avait déjà vanté la séduisante vivacité de ces statues ${ }^{36}$. Car, par l'habit du couronnement et grâce à une composition chromatique fidèle, on avait ajusté au plus ressemblant la présence durable du roi dans l'image de son éphémère apparition au cours du cérémoniel.

Au cours de son séjour au Palais de la Cité, l'empereur avait eu plusieurs occasions de contempler cet ensemble. La plus connue fut sans doute le banquet festif auquel les enlumineurs de l'exemplaire royal des Grandes Chroniques ont consacré l'une des rares miniatures de quasi pleine page ${ }^{37}$. Pendant le banquet, Charles IV ne fut pas seulement témoin d'une représentation scénique de la conquête de Jérusalem par Godefroy de Bouillon, mais put également voir de nombreux surtouts de table et notamment, dominant les têtes des convives, la généalogie monumentale des rois de France dont on avait pour l'occasion spécialement entouré les statues de tapisseries - «si bien ordenez et si à point mis », comme le remarquent expressément les Grandes Chroniques, « que les roys, qui sont de pierre tout autour, n'estoient point occupez ne empeschiez de veoir $»^{38}$.

33. $C f$. la représentation de la «Crucifixion du Parlement de Paris» (vers 1453-1455) au musée du Louvre qui concorde largement avec le modèle du portail tel que les sources écrites l'ont transmis ; voir pour ce tableau : Ph. LORENTZ, « À propos du "réalisme flamand" : La "Crucifixion du Parlement de Paris" et la porte du beau roi Philippe au Palais de la Cité », Cahiers de la Rotonde, 20, 1998, p. 101-124 ; ID., La crucifixion du Parlement de Paris, Paris, 2004 ; pour les sources écrites J. Guérout, «Le Palais de la Cité à Paris des origines à 1417 », op. cit., 2, 1950, p. 87-93.

34. J. GuÉRout, «Le Palais de la Cité à Paris des origines à 1417 », op. cit., p. 128-143; U. BENNERT, «Art et propagande politique sous Philippe IV le Bel : le cycle des rois de France dans la Grand'Salle du palais de la Cité », Revue de l'art, 97, 1992, p. 46-59.

35. Paris, BnF, Estampes, Vx 15, p. 269.

36. Jean de Jandun, Tractatus de laudibus Parisius, dans A. Le Roux De Lincy, L.-M. TisSerand éd., Paris et ses historiens aux XIV et XV siècles. Documents et écrits originaux recueillis et commentés, Paris, 1867, p. 32-79, ici p. 48.

37. Paris, BnF, ms. fr. $2813, \mathrm{f}^{\circ} 473 \mathrm{v}^{\circ}$; ici le texte fait mention expresse de la miniature : «[...] comme il est figuré en l'ystoire, ci après pourtraite et ymaginée. »

38. Chronique des règnes de Jean II et de Charles V, op. cit., vol. 2, p. 237. 
La visibilité jouait également un rôle déterminant au cours des services divins auxquels l'empereur assista dans la Sainte-Chapelle. D'après les Grandes Chroniques, l'espace avait été décoré et illuminé avec une telle majesté «que c'estoit belle et merveilleuse chose à veoir ${ }^{39}$. À l'inverse, l'empereur recherchait en petit comité la proximité directe, le toucher même des reliques de la Passion. C'est ainsi que Charles V le conduisit personnellement sur la tribune aux reliques :

Et quant il fu amont et le Roy ot ouverte la sainte chasse, le dit Empereur osta son chaperon et joint les mains, et comme en lermes fist là son oroison longuement, en tres grant devocion, et puis se fist soustenir et aporter baisier les saintes reliques. Et li monstra et devisa le Roy toutes les pieces qui sont en la dite chasse. Et après ce que les princes, qui avec lui estoient, orent baissié, le Roy tourna la dite chasse devers la chapelle, et laissa à garder ycelle les evesques de Beauvaiz et de Paris, revestus en pontifical de mictres et de crosses $^{40}$.

La signification majeure de l'ensemble de reliques ${ }^{41}$ qui y est conservé est illustrée avec force par un type iconographique présent dans l'enluminure qui figure la vue de la Grande Châsse ouverte avec tous ses reliquaires ${ }^{42}$ suivant le modèle bien connu des pieuses représentations des Arma Christi ${ }^{43}$, des instruments de la Passion. La vénération dont ces reliques faisaient l'objet constituait un commandement absolu de la piété envers la Passion auquel, suivant le modèle exemplaire du pieux monarque, obéissait saint Louis dans un manuscrit de La vie et les miracles de saint Louis de Guillaume de SaintPathus (vers 1330-1340) ${ }^{44}$ tout comme s'y soumettait Philippe VI dans le livre d'heures de Jeanne II de Navarre (vers 1336-1340) ${ }^{45}$.

De même, la main directive de Charles $\mathrm{V}$ désignant les reliques, dont l'empereur était le destinataire et le témoin dans la Sainte-Chapelle, suivait une attribution monarchique des rôles qui remontait à saint Louis, c'est-àdire à l'acheteur des reliques et au fondateur de leur culte. Dans un manuscrit probablement autographe des Chronica maiora de Mathieu Paris des années 1250 , ce rôle monarchique est préfiguré dans le texte comme dans l'image ${ }^{46}$. À travers le passage en revue des reliques sacrées ainsi proposé à l'empereur, Charles V donnait la manifestation évidente de ce sur quoi ne cessait pas

39. Ibid., p. 229.

40. Ibid., p. 233.

41. Le trésor de la Sainte-Chapelle, Paris, 2001 (catalogue de l'exposition de Paris).

42. Ibid., $\mathrm{n}^{\text {os }} 28-30$ et 71 .

43. $C f$. par exemple les Arma Christi dans le livre d'heures du Maréchal Boucicaut (vers 1408), Paris, Musée Jacquemart-André, ms. 2, $\mathrm{f}^{\mathrm{D}} 242$; voir Paris 1400. Les arts sous Charles VI, Paris, 2004 (catalogue de l'exposition de Paris), n 172.

44. Paris, BnF, ms. fr. 5716, $\mathrm{f}^{\circ} 67$; Le trésor de la Sainte-Chapelle, op. cit., $\mathrm{n}^{\circ} 24$.

45. Paris, BnF, ms. n. a. lat. 3145, $\mathrm{f}^{\circ} 150$; Le trésor de la Sainte-Chapelle, op. cit., $\mathrm{n}^{\circ} 46$.

46. Cambridge, Corpus Christi College, ms. 16, $\mathrm{f}^{\mathrm{o}} 141 \mathrm{v}^{\circ}$; S. LewIs, The Art of Matthew Paris in the Chronica Majora, Aldershot, 1987, p. 305-309 et 446-457. 
d'insister l'entourage de ses lettrés dans les traités de théologie politique: que le « roi très chrétien » était le magister sacrorum scriniorum, et que c'est pour cette raison que «doit estre le paÿs de France appellé la Saincte Terre, sur tous aultres princes seculiers, ce Roy et son peuple sont de Diex esleüs $\gg{ }^{47}$.

Après leur séjour dans le Palais de la Cité, le roi et l'empereur descendirent la Seine jusqu'au Louvre sur un navire d'apparat : " Ainsi arriverent au Louvre, et fu apporté le dit Empereur en sa chaiere, et le Roy estoit tousjours costé lui, jusques à ce qu'il fu dedenz le dit chastel, et li monstra et fist monstrer, au dehors et dedenz, le nouvel edifice que il y avoit fait, dont l'Empereur par semblant prenoit tres grant plaisir ${ }^{48}$. » À cette occasion, le roi n'a pas dû manquer de montrer également à l'empereur, à grand renfort d'explications, la célèbre Grande Vis. Comme il ressort de descriptions plus tardives, notamment dans l'Histoire et recherches des antiquités de la ville de Paris d'Henri Sauval (1724), sa façade visible offrait aux regards le cycle sculptuaire sans doute le plus ample de l'art de cour de Charles V. Il désignait à la vue de tous le couple royal au milieu des soutiens les plus importants de son pouvoir, c'est-à-dire les détenteurs des principautés apanagées ${ }^{49}$. Amputée de sa matérialité irrémédiablement perdue, la grande vis ne prend plus forme depuis que comme support de la puissance imaginative artistique d'un Viollet-le-Duc ou d'un Fédor Hoffbauer ${ }^{50}$.

L'itinéraire qu'emprunta ensuite l'empereur pour traverser les espaces de la représentation du pouvoir royal était également bordé de statues. Pendant son séjour dans l'Hôtel Saint-Pol ${ }^{51}$, l'église des Célestins située dans son voisinage direct n'aura pas non plus échappé à ses regards. Sur le portail de cette fondation royale, on pouvait voir l'effigie de Charles $\mathrm{V}$ en monarque tenu par les obligations de l'humilitas ${ }^{52}$. Pour se rendre à Vincennes, le cortège de la cour passa par la porte Saint-Antoine, c'est-à-dire, avant 13821383 environ, par la Bastille ${ }^{53}$ même. Là aussi, sur la façade orientale, le visiteur pouvait contempler les statues de son hôte comme autant de signes incontestables de son pouvoir. En effet, saint Antoine, représenté au centre de cet ensemble en patron des pauvres et des malades et en thaumaturge aux pouvoirs miraculeux, ne renvoyait pas seulement au devoir de miséricorde

47. [Évrard DE TRÉMAUGON] Le Songe du Vergier, édité d'après le manuscrit royal 19 C IV de la British Library par M. SCHNERB-Lièvre, 2 vol., Paris, 1982, vol. 1, p. 327 sq.

48. Chronique des règnes de Jean II et de Charles V, op. cit., vol. 2, p. 245.

49. Voir B. CARquÉ, Stil und Erinnerung. Französische Hofkunst im Jahrhundert Karls V. und im Zeitalter ihrer Deutung, Göttingen, 2004, p. 437-440.

50. E.-E. Viollet-Le-Duc, Dictionnaire raisonné de l'architecture française, op. cit., vol. V, p. 304 ; F. HoffBauer, Paris à travers les âges, op. cit., p. 327.

51. F. Bournon, «L'hôtel royal de Saint-Pol», Mémoires de la Société de l'Histoire de Paris et de l'Ile-de-France 6, 1879, p. 54-179.

52. B. Carqué, Stil und Erinnerung..., op. cit., p. 557 et fig. 130.

53. Voir à ce sujet les articles de A. ErLande-Brandenburg et N. Faucherre dans Sous les pavés, la Bastille. Archéologie d'un mythe révolutionnaire, Paris, 1989 (catalogue de l'exposition de Paris) ; N. Faucherre, «La Bastille, citadelle avant l'heure? », dans Fr. Pleybert dir., Paris et Charles V, op. cit., p. 85-90. 
et d'assistance du souverain mais incarnait en même temps le rappel du miracle royal de la guérison des écrouelles ${ }^{54}$.

C'est par la Tour du Village que le cortège royal fit enfin son entrée au château de Vincennes ${ }^{55}$ où il prit ses quartiers dans le donjon. Les niches aujourd'hui orphelines des deux édifices prouvent que l'empereur fut ici aussi confronté à une statuaire chargée de lui renvoyer la traduction visuelle de la conscience que pouvait avoir le roi de France de son propre pouvoir. Compte tenu du fait que les Grandes Chroniques font état d'une visite détaillée de l'ensemble du complexe architectural ${ }^{56}$, on est en droit de penser que l'on avait veillé, du côté de la puissance invitante, à ce que l'empereur fût en mesure de percevoir et de saisir l'architecture et les statues dans le sens souhaité. Les statues elles-mêmes y apportaient leur contribution en offrant au regard et dans un grand luxe de détails les regalia chargés de souligner le rang royal. Ainsi, sur le portail du Louvre, la statue de Charles V montraitelle le roi dans son costume original d'apparat tel qu'on peut le reconnaître, quant à l'habit et aux couleurs, sur une statue de saint Louis, provenant du château d'Enguerran de Marigny à Mainneville ${ }^{57}$, et également dans une miniature d'environ $1320^{58}$, cette fois encore pour les insignes.

On peut lire dans les pratiques socio-politiques de cette «visite d'État» des formes d'évolution des modes de réception qui n'ont pas abandonné au hasard la manière dont les lieux et les signes du pouvoir furent perçus et compris. Ce faisant, ces formes pouvaient s'appuyer sur des représentations et des attentes socialement déterminées, c'est-à-dire attachées à des groupes spécifiques: le concept de magnificentia définissait les monuments de cet art de cour comme autant de signes du soin apporté au bien commun et comme autant de signalements du déploiement des fastes du pouvoir ${ }^{59}$. En effet, l'éthique de majesté, en acte comme en esprit, trouvait son corrélat - parce que communiquée par les truchements de la représentation symbolique - dans la grandeur inscrite dans l'apparence projetée.

Cette image renvoyée était assurément avant tout destinée à l'espace public représentatif de la cour. Car, en dehors de sa dimension socio-politique géné-

54. B. CARQuÉ, Stil und Erinnerung, op. cit., en particulier p. 435 sq. avec fig. 132 et 133.

55. J. Сhapelot, Le château de Vincennes. Une résidence royale au Moyen Âge, Paris, 1994 ; ID. et É. Lalou dir., Vincennes aux origines de l'État moderne, op. cit.; Vincennes. Du manoir capétien à la résidence de Charles V (= Dossiers d'Archéologie 289, 2003/2004).

56. Chronique des règnes de Jean II et de Charles V, op. cit., vol. 2, p. 263 et 265.

57. Mainneville, Église Saint-Pierre; L'Art au temps des rois maudits. Philippe le Bel et ses fils, 1285-1328, Paris, 1998 (catalogue de l'exposition de Paris), $\mathrm{n}^{\circ} 51$.

58. Registre des ordonnances de l'Hôtel du roi, Paris, Archives nationales, JJ 57, f 20 ; La France de Saint Louis, Paris, 1970 (catalogue de l'exposition de Paris), nº 213.

59. Sur ce sujet complexe, voir entre autres B. Franke, «Magnifizenz. Die Tugend der Prachtentfaltung und die französische Kunst um 1400 », dans Th. SCHILP, B. Welzel dir., Dortmund und Conrad von Soest im spätmittelalterlichen Europa, Bielefeld, 2004, p. 141-161 ; W. ParavicinI, « Die zwölf < Magnificences > Karls des Kühnen », dans G. Althoff dir., Formen und Funktionen öffentlicher Kommunikation im Mittelalter, Stuttgart, 2001, p. 319-395. 
rale - c'est-à-dire l'obligation royale de largesse et d'assistance envers la communauté - la magnificentia possédait également des aspects bien plus exclusifs et socialement spécifiés. Elle formait une marque distinctive cruciale de l'univers de la haute-noblesse, marque dont l'accentuation respective se mesurait - comme on en trouve maintes fois la trace chez Froissart ou dans les Grandes Chroniques - selon l' «état» et le «degré» de «poissance » et de «rikèce» de ceux qui en étaient les porteurs ${ }^{60}$. De surcroît, les médias empruntés par la magnificentia étaient affectés d'une valeur prospective car, érigés en monuments efficaces pour le présent et pour l'avenir, ils devaient contribuer à l'inscription particulière du souvenir dans la durée, comme l'explique, à la cour de Charles V, Nicole Oresme : «C'est trop plus grant magnificence et meilleur chose de faire un temple ou un palais longuement durable de quoy il est aussi comme perpetuel memoire de celuy qui l'a fait et proffit au bien publique, que de faire une tele chose bele et jolie et de petite duree. Mais se elle estoit belle et durable, ce seroit tres bien. ${ }^{61}$

En dernier lieu, l'inscription dans l'espace public et l'inscription dans la durée conféraient également aux monuments une autorité et une crédibilité du plus haut degré. On peut s'en assurer par un procès dans lequel les «ymages» furent convoquées en témoignage à titre de preuves. En 1410, dressés les uns contre les autres sur la question de la possession de la vraie relique du crâne de saint Denis, les moines de Saint-Denis et les chanoines de Notre-Dame s'étaient retrouvés devant les grilles du Parlement de Paris. Au cours de l'échange des argumentations précédant le procès, le chapitre collégial prétendit que la calotte crânienne qui se trouvait en sa possession était bien la vraie relique, au titre que cette forme du martyre, et non pas la décapitation même, était prouvée par « de grans et anciens ymages de pierre et en lieux patens et publiques, au veu et sceu de tous ceulx qui l'ont volu veoir ${ }^{62} »$.

De tels modèles de perception et d'interprétation - entendons celui de la magnificentia comme celui de la légitimation acquise par la visibilité - marquent l'horizon élargi d'attributions de sens spécifiques qui pouvaient être saisies dans le concret des contextes d'action et des situations de réception d'une «visite d'État». C'est précisément dans ces instants fugaces d'une production active de sens spécifique et concrétisé en situation que l'histoire de l'art semble pouvoir s'approcher de la réalité historique des productions architecturales et iconographiques, plus en tout cas que par le biais de la simple reconquête de leur visibilité perdue.

(Traduit de l'allemand par Pierre MonNET)

60. Voir les exemples dans B. CARQuÉ, Stil und Erinnerung, op. cit., p. 450 sq.

61. Nicole Oresme, Le Livre de Ethiques d'Aristote, éd. A. D. Menut, New York, 1940, p. 246.

62. H. Fr. Delaborde, «Le procès du chef de saint Denis en 1410 », Mémoires de la Société de l'Histoire de Paris et de l'Ile-de-France 11, 1884, p. 297-409, ici p. 399. 
Bernd Carqué, Baurat-Gerber-Straße 18, D-37073 Göttingen

\section{«Paris 1377-78 ». Un lieu de pouvoir et sa visibilité entre Moyen Âge et temps présent}

Les politiques de représentation visuelle du pouvoir à travers les monuments de Paris à la fin du Moyen Âge ont attiré l'attention des chercheurs dans les dernières décennies. La plupart des études se sont portées sur la simple apparence de constructions comme le Palais de la Cité ou encore le Château du Louvre. Leurs structures architecturales et leurs éléments formels ont été interprétés comme des expressions authentiques du pouvoir royal et comme des signes architectoniques permettant de rendre visible la royauté. Dans la mesure où les bâtiments pris en considération ont été détruits, ces interprétations iconographiques reposent sur des sources qui sont elles-mêmes problématiques. Comme le montre cet article, les représentations contemporaines des résidences royales comme celles qu'on trouve dans les miniatures des fameuses «Très Riches Heures du duc de Berry » sont une base douteuse pour reconstituer des monuments disparus et leur signification formelle, en raison de la variabilité et des discordances qu'elles présentent. À cet égard, elles sont aussi incertaines que les reconstructions modernes. Une solution qu'on imagine rarement pour résoudre ce problème de la disparition physique des bâtiments consiste à se demander de quelle manière les édifices royaux ont été utilisés, montrés et expliqués. C'est sous cet aspect que l'article réexamine la célèbre visite de l'empereur Charles IV à Paris en 1377-78. Un ouvrage récent s'est intéressé à la fois à l'aspect cérémoniel de la visite et à l'usage de signes éphémères comme les armoiries, les livrées ou les devises. En revanche, on ne sait presque rien du rôle que jouèrent les monuments durables dans le cadre de ces cérémonies. Le but de cet article est de montrer que la signification des bâtiments royaux a été établie et s'est concrétisée dans le cadre de situations de perception bien organisées.

Paris tardo-médiéval - lieux de pouvoir - Charles V - architecture - cérémonial - perception - édifices royaux

\section{Paris 1377-78. Places of Power and the Problems of Visibility between the Middle Ages and Present Time}

The politics of visual representation through monuments in late medieval Paris have received the broadest scholarly attention during the last decades. Most of this art historical interest, however, has been directed towards the mere appearance of buildings such as the Palais de la Cité or the Château du Louvre. Their physical structures and formal elements have been interpreted as genuine expressions of royal power and as architectonic signs allowing a visualization of kingship. Insofar as the buildings under consideration have been destroyed, such iconographical interpretations are based on problematic sources. This article shows how contemporary representations of royal palaces such as the calendar miniatures in the famous "Très Riches Heures du duc de Berry » are a doubtful basis for the reconstruction of lost monuments and of their former signification because of their variability and discrepancies. In this respect they are as uncertain as modern reconstructions are. 
A hardly considered solution to the problem of missing physical evidence will be to ask in what ways royal buildings have been used, shown and explained. It is from that angle that this article re-examines the well known visit of the Holy Roman Emperor Charles IV in Paris 1377-78. Recent work has investigated both the ceremonial performance of the visit and the use of ephemeral signs such as armorial bearings, liveries or devices. By contrast, almost nothing is known about the part played by long-lasting monuments in connexion with the ceremonies. The purpose of this contribution is to demonstrate that the signification of royal buildings has been established and concretized in well organized situations of perception.

Late medieval Paris - Charles V - architecture - ceremonial - places of power - royal buildings - perception 